\title{
GPPS-BJ-2019-116
}

\section{A MESHLESS MODEL FOR CAVITY NOISE PREDICTION BASED ON VORTEX SOUND METHOD}

\author{
Zengze Yu \\ School of Mechanical Engineering \\ Shijiazhuang Tiedao University \\ yuzz6310@163.com \\ Shijiazhuang, Hebei province, China
}

\author{
Zhiliang Hong \\ The Airworthiness College, \\ Civil Aviation University of China \\ hongzl@stdu.edu.cn \\ Tianjin, PR China
}

\begin{abstract}
A meshless method for predicting cavity noise is developed by using the discrete vortex method and the vortex theory. Firstly, the discrete vortex sound method is used to calculate the flow field of the cavity under uniform grazing flow, and the key parameters such as the strength, position and velocity of the point vortices are obtained. Then, based on vortex sound theory, the sound pressure of point vortex in the free space is obtained, and the time-domain boundary element method is introduced to simulate the scattering effect of the cavity surface. In the end, the spectrum of the sound pressure is analysed. This meshless method has high computational efficiency, and can obtain the key characteristics of the flow field and sound field simultaneously. It can improve the basic understanding of the vortex sound mechanism of cavity noise. At the same time, it provides a reliable model method with satisfactory efficiency, which can be readily extended to the corresponding engineering applications.
\end{abstract}

\section{INTRODUCTION}

With the increase of the speed of high-speed trains, the noise pollution problem gets more and more attention from the public, which has become an important limiting factor for the development of high-speed trains. In general, the main noise sources of high-speed trains include traction noise, wheel-rail noise and aerodynamic noise, and the last component will become dominant when the vehicle speed reaches $300 \mathrm{~km} / \mathrm{h}$ (Thompson et al., 2015). Among the various aerodynamic noise sources, the noise from the innercoach space (which can be regarded as a rectangular cavity) is very complicated. When the grazing flow passes the surface of the inner-coach space of the running train, selfexcited oscillation (acoustic resonance (Gloerfelt, 2009)) can occur, due to the interaction between the shear flow outside the cavity and the acoustic wave in the cavity. This is a complex coupling phenomenon between the flow and the sound field. It has attracted enough attention from designers in many engineering applications, such as inner-coach space of high-speed train automobile sunroof, military bomb bay and wheel well of civil craft, therefore researches are greatly motivated to explore the underlying mechanism of this phenomenon.

It is generally believed that the physical mechanism of acoustic resonance is that separation occurs when the flow reaches the sharp leading edge of the cavity, forming a shear layer with inherent instability. Frequency lock-in will occur when the oscillation frequency of the shear layer is consistent with the natural acoustic mode frequency of the surrounding cavity. The oscillation frequency of the shear layer does not change with the flow velocity within a certain speed range. At the same time, this phenomenon exacerbates the energy exchange between vortex and sound, resulting in large unsteady pressure fluctuation. In short, the excitation of the shedding vortex is the main cause of the acoustic resonance of the cavity. There are four main stages in the process of cavity noise generation: (1) due to the instability of the shear layer, the induced vortex disturbance is continuously increased, forming a non-uniform eddy current motion; (2) the vortex and rigid wall interaction generates pressure disturbance, causing the pressure field to be uneven; (3) The pressure disturbance propagates forward in the low velocity region of the cavity, and radiates energy to the space in the form of noise outside the cavity; (4) under the influence of pressure disturbance, the vortex shedding at the leading edge of the cavity is enhanced, and the initial shear layer disturbance is further increased.

The semi-empirical equation first proposed by Rossiter (1964) relies on the selection of empirical constants. Most of these methods (Bilanin et al., 2012; Heller et al., 1971; Tam et al., 1978) can only estimate the modal frequencies of all possible oscillations in a simple cavity. However, such models cannot predict the amplitude of the oscillating mode and exhibit the mechanism of the acoustic resonance of the cavity. Although, the numerical method, such as DNS and LES (Colonius et al., 1999; Rowley, et al., 2002; Shieh et al., 
2013; Gloerfelt et al., 2002a; Gloerfelt et al., 2013b), performs excellent in illustrating the details of flow and sound field, there is a huge difference between the scale of the physical variations in the flow and sound field, resulting in such methods being terribly time-consuming.

Aiming to the physical essence of cavity noise, the object of present study is establishing a time-domain meshless model with high computational efficiency and enough accuracy to give a detailed description of the distribution of flow field. It can also calculate some parameters of the sound field, such as the sound pressure level and the sound pressure frequency at a certain point.

\section{METHODOLOGY}

\section{Discrete vortex model for calculating the flow field of a cavity}

This work is based on a general two-dimensional cavity of length $2 s$ and depth $d$ as shown in figure 1(a). For the solution of the cavity flow field, this paper refers to Hardin's (1977) discrete vortex method, and only the main steps are briefly explained here.

In the case of a low flow velocity, the shedding vortex is mainly separated from the leading edge of the cavity. A uniform flow with speed $U_{g}$ grazingly passes over the cavity. Assume that the flow field is non-viscous, and because the Mach number is low, it can be assumed that the flow field is incompressible. So the discrete vortex method can be applied to solve the flow field. It is supposed that a nascent vortex is generated at the leading edge of the cavity at each time step, and its intensity is determined by the Kutta condition. The strength of the point vortex will gradually decrease in consideration of the actual physical condition. This model refers to the practice of Kiya et al. (1982) and introduces equation (1) to describe the variation of the strength of the shedding vortex over time:

$$
\frac{\Gamma(t)}{\Gamma_{0}}=1-\exp \left(-\frac{\alpha^{2} \operatorname{Re}}{2 U_{g} t / s}\right)
$$

where, $\Gamma_{0}$ is the initial strength of the point vortex; $\Gamma(t)$ is the intensity of the point vortex at a certain moment $t ; \alpha$ is a constant, related to the type of flow; $\operatorname{Re}=2 U_{g} s / \nu$ is the Reynolds number, where $U_{g}$ is the component of the incoming flow in the $x$ direction, $s$ is the half length of the cavity, $v$ is the kinematic viscosity coefficient, take $\alpha^{2} \operatorname{Re}=24$ here. It should be pointed out that if $\alpha$ is regarded as the distance from the center of the point vortex, then equation (1) is the solution of the N-S equation of the line viscosity vortex.

The trajectory of the point vortex is given by Kelvin theory:

$$
\frac{d z_{n}}{d t}=u\left(z_{n}\right)
$$

where $z_{n}$ is the position of the nth point vortex; $u$ is the speed of the point vortex.

\section{Schwartz-Christoffel transformation}

To facilitate modelling process, a Schwartz-Christoffel transformation is applied to project the physical flow region $Z$ into the upper half-plane $W$ of the complex variable. The cavity wall is transformed into the real axis of the $W$-plane, and the four corners on the wall are transformed into $( \pm a, 0)$ and $( \pm 1,0)$, respectively, as shown in figure 1 . The SchwartzChristoffel transformation is:

$$
\mathrm{z}=\frac{s \cdot E\left(\sin ^{-1} \lambda \backslash \sin ^{-1}(1 / a)\right)}{E\left(1 \backslash a^{2}\right)}
$$

where $z=x+i y, \lambda=\zeta+i \eta, E(\cdot \cdot \cdot)$ and $E(\cdot)$ are the incomplete and complete elliptical integral of the second kind with complex argument, respectively (Abramowitz $\mathrm{M}$ et al., 1964). Parameter $a$ is a constant associated with the depth to length ratio of the cavity, as shown in figure 2.

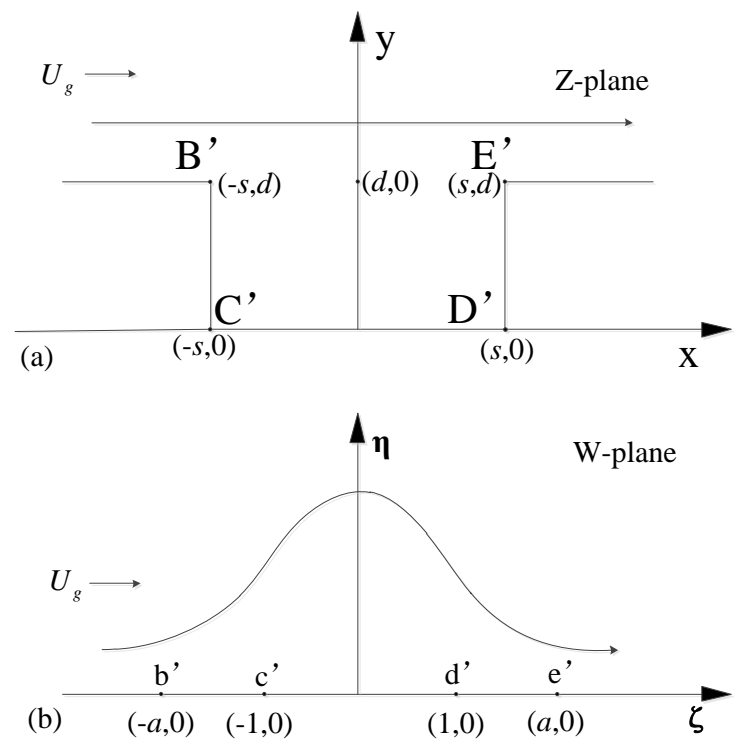

Figure 1 Schematic Diagram of Conformal Transformation

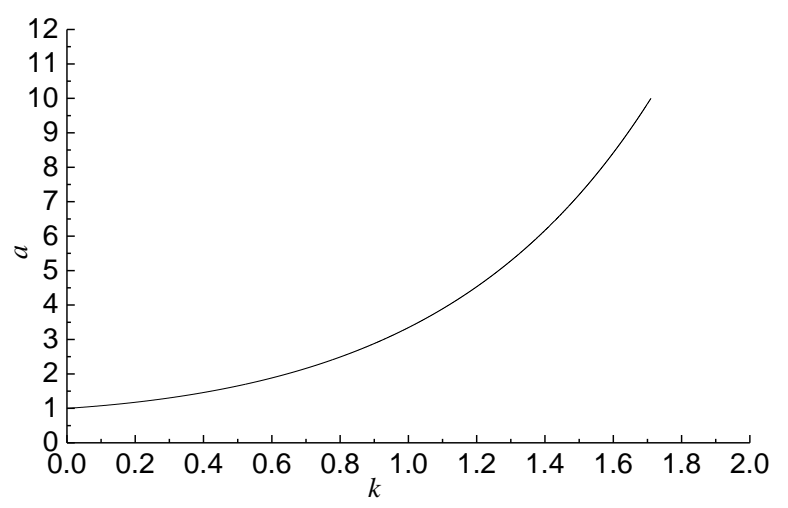

\section{Figure 2 the Parameter $a$ as a Function of Parameter $k$, where $k=d / s$}

\section{The speed of point vortex}

According to the potential flow theory, in the transform domain, the total potential function of the flow field can be 
obtained by adding the potential functions induced by the tangential flow and point vortices:

$$
\begin{aligned}
W(\lambda)= & \frac{s \cdot U_{g}}{a \cdot E\left(1 / a^{2}\right)} \lambda+ \\
& \sum_{j=1}^{\infty} \frac{\Gamma_{j}}{2 \pi i}\left[\ln \left(\lambda-\lambda_{j}\right)-\ln \left(\lambda-\lambda_{j}^{*}\right)\right]
\end{aligned}
$$

where $\Gamma_{j}$ and $\lambda_{j}$ are the strength and coordinates of the jth point vortex, respectively, * indicates conjugate. Therefore, for the velocity $u\left(z_{k}\right)$ of the kth point vortex in the physical domain, it can be obtained by the differential velocity function $W_{z_{k}}(z)$ :

$$
\begin{array}{r}
u\left(z_{k}\right)=\frac{d W_{z_{k}}(z)}{d z}=\sqrt{\frac{\lambda_{k}^{2}-1}{\lambda_{k}^{2}-a^{2}}}\left[U_{g}+\sum_{\substack{j=1 \\
j \neq k}}^{\infty} \frac{\Gamma_{j}}{2 s \pi i} \frac{a \cdot E\left(1 / a^{2}\right)}{\lambda_{k}-\lambda_{j}}-\right. \\
\left.\sum_{j=1}^{\infty} \frac{\Gamma_{j}}{2 s \pi i} \frac{a \cdot E\left(1 / a^{2}\right)}{\lambda_{k}-\lambda_{j}^{*}}+\frac{\Gamma_{k}}{4 \pi i} \frac{a \cdot E\left(1 / a^{2}\right) \cdot \lambda_{k} \cdot\left(1-a^{2}\right)}{s \cdot\left(\lambda_{k}^{2}-1\right)\left(\lambda_{k}^{2}-a^{2}\right)}\right]
\end{array}
$$

where $z_{k}$ and $\lambda_{k}$ are the positions of the kth point vortex in the physical domain and the computational domain, respectively.

It can be seen that if the position of each point vortex at time $t$ is known, the velocity of the point vortex can be calculated by equation (5). And then the numerical method can be used to solve equation (2) to obtain the position of each point vortex at the next moment, that is, the strength distribution in the flow field.

\section{Strength and position of the nascent vortex}

During the calculation process, a point vortex is generated at the upper corner of the leading edge of the cavity at each time step. When the displacement of other point vortices in the flow field is calculated numerically, the new point vortex convects downstream and is connected to the corner by the feeding sheet, as shown in figure 3 . Until the end of the time step, the new point vortex will truly leave the upper corner of the front edge of the cavity and become a free point vortex.

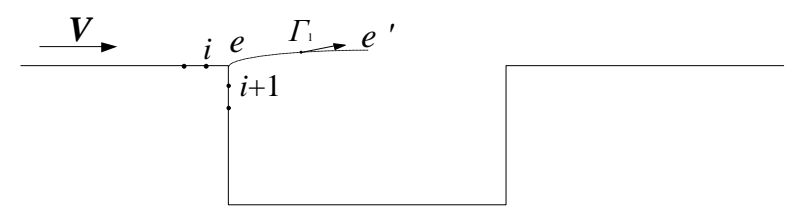

\section{Figure 3 Shedding Vortex Model at the Corner of the Leading Edge of the Cavity}

In the calculation process, if the selected time step $\Delta t$ is relatively small, the corresponding length of feeding sheet will be relatively small, so it can be approximated by a point vortex at the center of $e e^{\prime}$. In addition, the velocity of point $e$ can be expressed as the vector sum of the two-point velocity adjacent to the orthogonal solid wall. Therefore the position of the new vortex can be expressed as:

$$
z_{1}=z_{e}+\frac{1}{2}\left(u_{i}+u_{i+1}\right) \Delta t
$$

where $z_{1}$ is the new vortex position; $z_{e}$ is the position of the upper corner of the leading edge of the cavity; $u_{i}$ and $u_{i+1}$ are the flow field velocities at the ith and $i+1$ th corner points, respectively.

The strength of the nascent vortex is determined after obtaining the position of the new vortex. When solving the velocity according to equation (5), it should be noted that the corner point is a singular point, which corresponds to the infinity flow velocity of the inviscid flow occurs at a sharp point. In order to comply with the actual physical situation, the singularity should be removed, which requires in the transform domain:

$$
\frac{d W}{d \lambda}=0, \lambda=-a
$$

Thus, for the shedding vortex at the corner of the front edge of the cavity, the intensity of the new point vortex is:

$$
\Gamma_{1}=-\frac{\left(a+\xi_{1}\right)^{2}+\eta_{1}^{2}}{\eta_{1}}\left[\frac{\pi \cdot s \cdot U_{g}}{a \cdot E\left(1 / a^{2}\right)}+\sum_{j=2}^{\infty} \frac{\Gamma_{j} \eta_{j}}{\left(a+\xi_{j}\right)^{2}+\eta_{j}^{2}}\right]
$$

\section{Point vortex generation model based on vortex theory}

This part refers to the work of Hong et al. (2015) and is only briefly introduced here. The sound pressure of point vortices in the free space is calculated firstly. Since the surface of the cavity has a reflection effect on the sound, it is realized by the time domain boundary element method.

\section{Point vortex sounds in free space}

Because this work is based on the key factors of vortex to describe the flow field, it is more convenient to use Howe's vortex theory to solve the sound field than the traditional Curle's (1955) method. In addition, the vortex theory can more intuitively indicate the transformation relationship between the flow field and the sound field (Jing et al., 2002).

Since the point vortex model is established under conditions of low Mach number, inviscid, and isentropic flow, the Howe (2003) vortex equation is:

$$
\left(\frac{1}{c^{2}} \frac{\partial^{2}}{\partial t^{2}}-\nabla^{2}\right) \frac{p}{\rho}=\nabla(\omega \times v)
$$

where $p$ is the sound pressure; $\rho$ is the density; $\omega$ is the rotation of the flow field; $v$ is the airflow velocity.

If the observation point is $\boldsymbol{x}=\left[x_{1}, x_{2}\right]$, the sound source point is $\boldsymbol{y}=\left[y_{1}, y_{2}\right]$, and the point vortex position is $\boldsymbol{s}$ $=\left[y_{1 n}, y_{2 n}\right]$. In the two-dimensional flow field, the pressure $p_{n}$ produced by the nth point vortex can be expressed as: 


$$
\begin{aligned}
\frac{p_{n}}{\rho} & =\int_{0}^{t} \int_{S^{\prime}} \nabla \cdot\left(\omega_{n} \times \boldsymbol{v}_{n}\right) G d S d \tau \\
& =-\int_{0}^{t} \int_{S^{\prime}}\left(\omega_{n} \times \boldsymbol{v}_{n}\right) \cdot \nabla G d S d \tau \\
& =\left.\int_{0}^{t}\left(\Gamma_{n} v_{n y} \frac{\partial G}{\partial y_{1}}-\Gamma_{n} v_{n x} \frac{\partial G}{\partial y_{2}}\right)\right|_{\left(y_{1 n}, y_{2 n}, \tau\right)} d \tau
\end{aligned}
$$

where $S^{\prime}$ is the area of the area including the sound source; $\boldsymbol{\omega}_{n}=\Gamma_{n} \boldsymbol{k} \delta\left(x-x_{n}(\tau)\right) \delta\left(y-y_{n}(\tau)\right)$ is the strength; $\delta(\cdot)$ is the Dirac delta function; $\boldsymbol{v}_{n}=\boldsymbol{i} v_{n x}+\boldsymbol{j} v_{n y}$ is the point vortex velocity; $\boldsymbol{i}, \boldsymbol{j}$ and $\boldsymbol{k}$ are the unit vectors in the $\boldsymbol{i}, \boldsymbol{j}$ and $\boldsymbol{k}$ directions, respectively; $G$ is the Green's function.

In two-dimensional space, the expression of the Green's function is:

$$
G(\boldsymbol{x}, t / \boldsymbol{y}, \tau)=\frac{c H[c(t-\tau)-R]}{2 \pi \sqrt{c^{2}(t-\tau)^{2}-R^{2}}}
$$

where $c$ is the speed of sound; $R=\|\boldsymbol{x}-\boldsymbol{y}\|=\sqrt{\left(x_{1}-y_{1}\right)^{2}-\left(x_{2}-y_{2}\right)^{2}} \quad$ is the distance between the source point and the field point; $H$ is the Heaviside function.

Substituting the equation (11) into the equation (10), the following expression is finally obtained:

$$
\begin{gathered}
\frac{p_{n}}{\rho}=\int_{0}^{t} \Gamma_{n}\left(v_{n x} \frac{\partial R_{n}}{\partial y_{2}}-v_{n y} \frac{\partial R_{n}}{\partial y_{1}}\right) \times \\
\frac{c(c(t-\tau)-R) H(c(t-\tau)-R)}{2 \pi \sqrt{\left[c^{2}(t-\tau)^{2}-R_{n}^{2}\right]^{3}}} d \tau+ \\
\int_{0}^{t} \frac{\partial\left[\Gamma_{n}\left(v_{n x} \frac{\partial R_{n}}{\partial y_{2}}-v_{n y} \frac{\partial R_{n}}{\partial y_{1}}\right)\right]}{\partial \tau} . \\
\frac{H(c(t-\tau)-R)}{2 \pi \sqrt{\left[c^{2}(t-\tau)^{2}-R_{n}^{2}\right]^{3}}} d \tau
\end{gathered}
$$

Thus, for all the point vortices in the flow field, there is:

$$
p=\sum_{n=1}^{N} p_{n}
$$

where $N$ is the total number of point vortices in the flow field.

\section{Effect of cavity surface on sound field}

Since the previous solution to the sound field is based on the Green's function of free space, the reflection of the acoustic surface on the cavity surface needs to be considered in the calculation of the sound field. This part needs to be implemented by means of the time domain boundary element method.

Considering the calculation area of this work, the time domain boundary integral form of the two-dimensional wave equation can be obtained without the initial sound field (Mansur, 1983):

$$
\begin{gathered}
c_{s}(Q) p(Q, t)=\int_{0}^{t} \int_{\Pi} \frac{\partial p}{\partial \boldsymbol{n}} \cdot \frac{c H(c(t-\tau)-R)}{2 \pi \sqrt{c^{2}(t-\tau)^{2}-R^{2}}} d \boldsymbol{\Pi} d \tau+ \\
\int_{0}^{t} \int_{\Pi} \frac{\partial R}{\partial \boldsymbol{n}}\left\{p \frac{c(c(t-\tau)-R) H(c(t-\tau)-R)}{2 \pi \sqrt{\left[c^{2}(t-\tau)^{2}-R^{2}\right]^{3}}}+\right. \\
\left.\frac{\partial p}{\partial \tau} \cdot \frac{H(c(t-\tau)-R)}{2 \pi \sqrt{c^{2}(t-\tau)^{2}-R^{2}}}\right\} d \boldsymbol{\Pi} d \tau+ \\
\int_{0}^{t} \int_{S} \gamma G d S d \tau
\end{gathered}
$$

where $c_{s}(Q)$ is a constant depending on the position of wave front; $\gamma=\nabla\left(\omega_{n} \times v_{n}\right)$ is a single-point vortex source term. $\boldsymbol{\Pi}$ is the boundary of the calculation domain $S ; \boldsymbol{n}$ is the out-of-boundary normal vector; $Q$ is the field point. It should be noted that the last item on the right side of the equation is the point vortex item.

\section{NUMERICAL SOLUXTION PROCESS}

\section{Solution of cavity flow field}

When using discrete vortices to solve the results of a cavity flow field, the calculation steps are as follows:

a. At the mth time step, use equation (6) to calculate the position of the new point vortex at the upper corner of the leading edge of the cavity;

b. Solve the velocity of other point vortices using equation (5);

c. Using the fourth-order Runge-Kutta method to solve the equation (2), the positional parameters of other point vortices in the flow field are obtained;

d. Using the Kutta condition (i.e. equation (8)) to give the strength of the new point vortex, and obtain the strength parameters of all other point vortices according to equation (1);

e. Update the time step $t_{m+1}=t_{m}+\Delta t$ and repeat the above steps.

When the discrete vortex method is used to calculate the flow field vortex, as time advances, the number of vortices in the flow field of the cavity will increase more and more, and the corresponding calculation time will become larger and larger. In actual physics, the point vortices in the flow field will move downstream as time passes, and the strength will eventually decay to zero due to the presence of viscosity. In order to better conform to the physical reality, the vortex attenuation and the vortex shedding method are used here to control the point strength and the number of point vortices.

\section{Solution of cavity sound field}

\section{Solution of Point Vortex Sounding Model}

When using the discrete vortex method to calculate the cavity flow field, the strength, velocity and position of the point vortex in each time step are obtained. According to equation (12), the radiation sound pressure of a single point 
vortex can be calculated. For the solution method of the calculation equation (14), there is a specific derivation process in Hong et al.'s (2015) work, which will not be described here. Finally, the sound pressure of the nth point vortex at time $t_{n}$ can be obtained.

$$
\frac{p_{n}\left(t_{n}\right)}{\rho}=\sum_{m=1}^{n-1} \frac{p_{n}\left(t_{m}\right)}{\rho}
$$

\section{Boundary Element Method for Solving Vortex Equation}

When solving the sound field with the time domain boundary element method, the main control equation (16) is rewritten into the following format (Mansur, 1983):

$$
\begin{aligned}
c_{s}(Q) p(Q, t) & =\int_{0}^{t} \int_{\Pi} \boldsymbol{b}(Q, t) G d \boldsymbol{\Pi} d \tau+ \\
& \int_{0}^{t} \int_{\Pi} \frac{\partial R}{\partial \boldsymbol{n}}\left(p B+\frac{\partial p}{\partial \tau} \cdot \frac{1}{c} \cdot G\right) d \boldsymbol{\Pi} d \tau+S_{0}
\end{aligned}
$$

In the above equation

$$
\left\{\begin{array}{l}
\boldsymbol{b}(Q, t)=\frac{\partial p(Q, t)}{\partial \boldsymbol{n}} \\
B=\frac{c(t-\tau)-R}{2 \pi \sqrt{c^{2}(t-\tau)^{2}-R^{2}}} H(c(t-\tau)-R) \\
S_{0}=\int_{0}^{t} \int_{S} \gamma \frac{c}{2 \pi \sqrt{c^{2}(t-\tau)^{2}-R^{2}}} H(c(t-\tau)-R) d S d \tau
\end{array}\right.
$$

The traditional collocation point method is used to discretize the equation, and finally:

$$
\begin{aligned}
c_{s}(Q) p_{i}^{n}+ & \sum_{j=1}^{J} H_{i j}^{n n} p_{j}^{n}-\sum_{j=1}^{J} \boldsymbol{D}_{i j}^{n n} b_{j}^{n}= \\
& \sum_{m=1}^{n-1} \sum_{j=1}^{J} D_{i j}^{n m} \boldsymbol{b}_{j}^{m}-\sum_{m=1}^{n-1} \sum_{j=1}^{J} H_{i j}^{n m} p_{j}^{m}+s_{i}^{n}
\end{aligned}
$$

In the above equation,

$$
\left\{\begin{array}{l}
H_{i j}^{n m}=-\int_{\Pi} \frac{\partial R}{\partial \boldsymbol{n}} \eta_{j}(Q) \int_{0}^{t_{n}}\left(\varphi^{m}(\tau) B+\frac{1}{c} \frac{d \varphi^{m}(\tau)}{\partial \tau} G\right) d \tau d \Pi \\
\boldsymbol{D}_{i j}^{n m}=\int_{\Pi} \zeta_{j}(Q) \int_{0}^{t_{n}} \theta^{m}(\tau) G d \Pi d \tau \\
s_{i}^{n}=\int_{0}^{t_{n}} \int_{S} \gamma G d S d \tau \\
p_{j}^{m}=p\left(Q_{j}, t_{m}\right) \\
\boldsymbol{b}_{i}^{m}=b\left(Q_{j}, t_{m}\right)
\end{array}\right.
$$

Thus, if the boundary conditions at each node at time $t_{n}$ are known, the sound pressure and the normal sound pressure gradient values at all nodes can be solved according to equation (16).

\section{RESULTS AND DISCUSSION}

A cavity with a depth to length ratio of $1: 2.35$ is considered. And the cavity length is $120 \mathrm{~mm}$, as shown in figure 4. It was placed in a flow field with a velocity of 39 $m / s$.

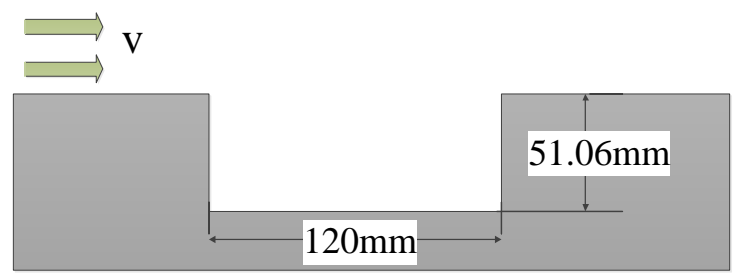

\section{Figure 4 Schematic Diagram of the Cavity in the Flow Field}

\section{Flow field result}

It should be noted that we adopt two kinds of methods to control the number of point vortices and so is the computational time within acceptable bounds. One is attenuating the strength of vortex through equation (1), and the other is discarding the vortices that are convected more than $10 s$ far away from the leading edge corner. The two methods adopted in this paper to control the number of point vortices in the flow field will discard the point vortices satisfying the conditions, which has little effect on the results. Through plenty of numerical experiments, the time step $\Delta t$ used in this work is set to be $3 \times 10^{-5} \mathrm{~s}$. Consequently, there are about 400 vortices in the flow field, after the wake flow is fully developed.

First, the variation of the strength of the nascent vortex over time is given, as shown in figure 5. Obviously, it is oscillating periodically. The period corresponds to the inherent shedding frequency of the vortex at the leading edge of the cavity. After FFT analysis, the basic frequency component is $175 \mathrm{~Hz}$, as shown in figure 6 .

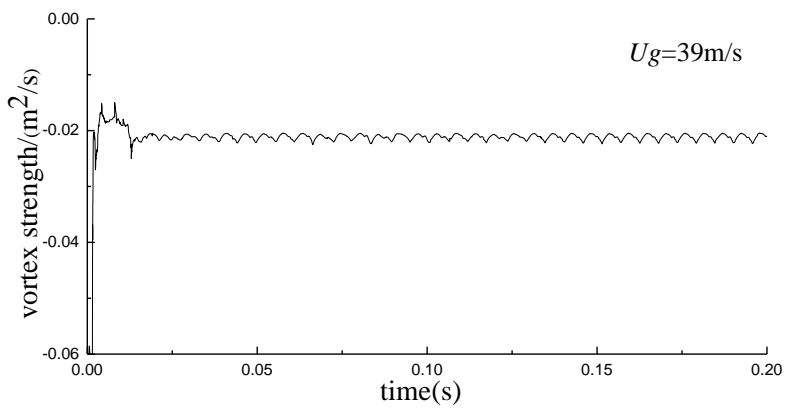

Figure 5 the Variation of Nascent Vortex Strength

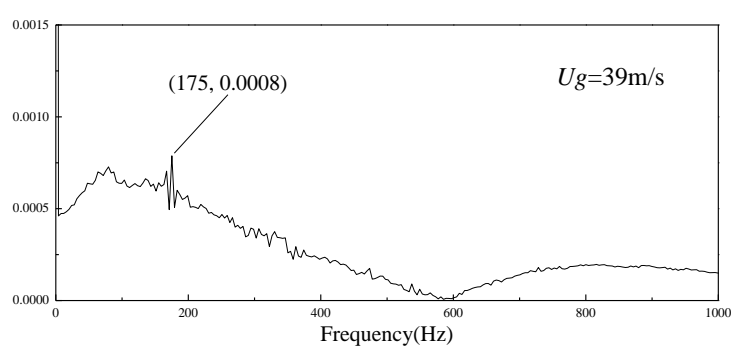

Figure 6 Shedding Frequency of Cavity Vortex 
For comparison, spectra of the strength of the cavity vortex at different speeds with time are also illustrated, as shown in figure 7.

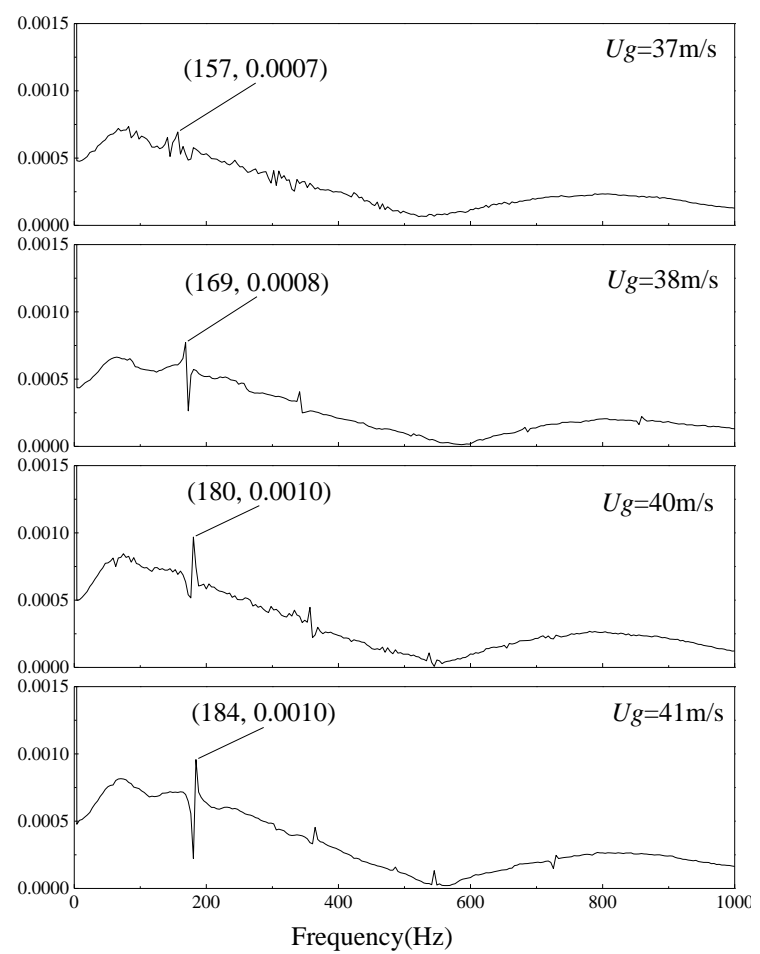

Figure 7 Shedding Frequency of Cavity Vortex at Different Inflow Speeds

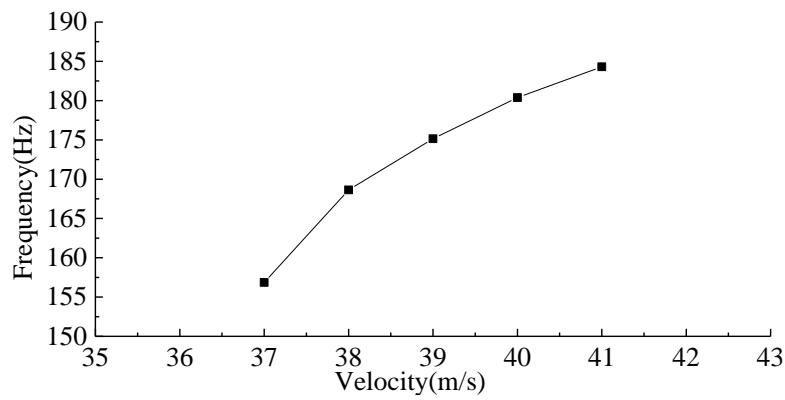

Figure 8 the Variation of the Shedding Vortex Frequency with the Flow Velocity

As the speed increases, the frequency of cavity vortex shedding increases and the law of variation remains substantially linear.

The distribution of point vortices in the flow field of the cavity is given below, as shown in figure 9 and figure 10 .

First, in figure 9, the initial flow field evolution of the cavity is shown. The shed vortices from the leading edge of the cavity forms a shear layer in the grazing flow and rolls up into a vortex cloud, while convecting downstream and eventually hitting the trailing edge of the cavity.

After a period of time, the flow field of the cavity is fully developed, and a vortex cluster with periodic evolution is formed at this time. The vortex evolution process in one cycle is given here, as shown in figure 10. It can be seen that at time $t=T / 4$, the vortex begins to roll up. At the time of $t$ $=T / 2$ and $t=3 T / 4$, the vortex gradually becomes larger and convects downstream under the influence of the mainstream. At time $t=T$, the vortex impinges the trailing edge of the cavity and then dissipates, while a new vortex starts to roll up. It can be seen that it is due to the oscillation of the shear layer that the vortex is continuously formed.

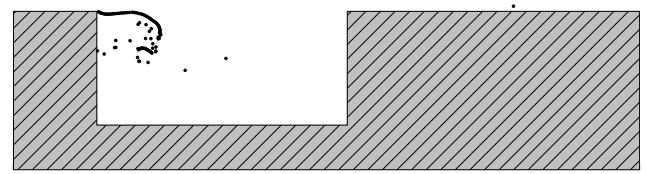

(a)

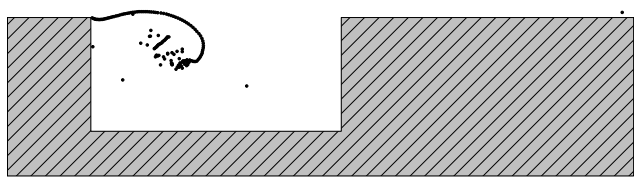

(b)

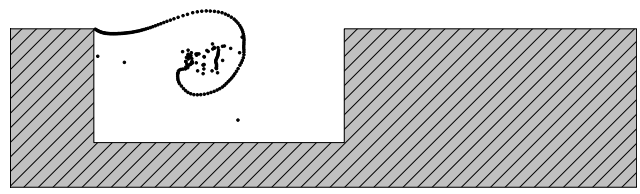

(c)

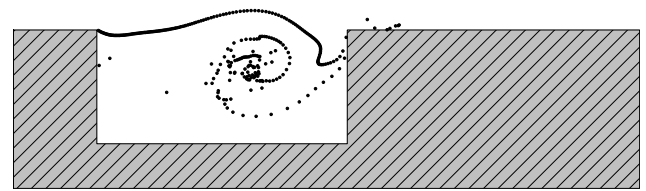

(d)

Figure 9 Evolution of Initial Flow Field of Cavity
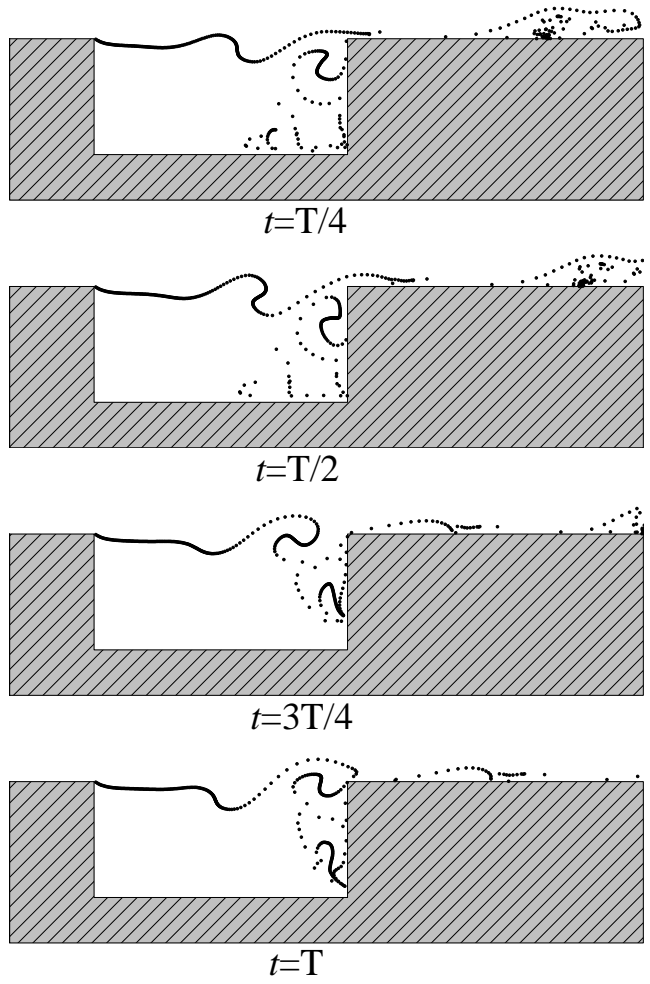

Figure 10 Schematic Diagram of the Evolution of Shedding Vortex in One Cycle 
When the flow velocity is low, the flow field flow type in the cavity is divided into a shear layer mode and a tail vortex mode (Grace, 2001). From figure 7, it can be seen that the flow filed flow type at this time is the shear layer mode.

\section{Sound field result}

When simulating the surface scattering of a cavity using a two-dimensional boundary element method, it is necessary to construct an auxiliary boundary that is enclosed with the surface of the cavity. The calculation domain is shown in figure 11.

The auxiliary boundary of the surface of the cavity is introduced only to satisfy the algorithm requirements of the boundary element, and it is not necessary to discretize it, but only the boundary of the cavity surface is discretized. The schematic diagram of the boundary conditions of the calculation region used in this work is also shown in figure 11.

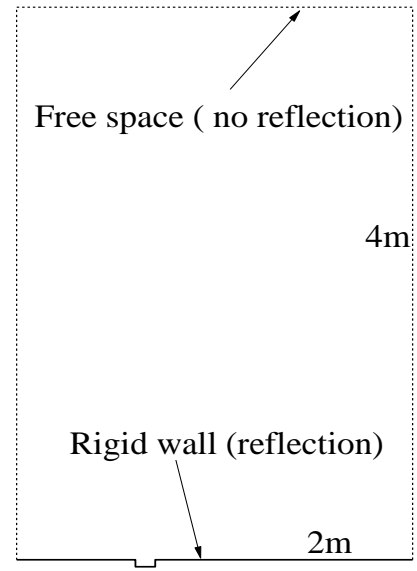

\section{Figure 11 Calculation Domain and Boundary Conditions}

In order to ensure the stability and accuracy of the time domain boundary element algorithm, it is necessary to control the step size of time and space. After a large number of numerical calculations, the boundary element length and time step selected as $0.02 \mathrm{~m}$ and $3 \times 10^{-5} \mathrm{~s}$, respectively, and the total number of boundary elements is 78 .

This paper selects an observation point $(x=0.5 \mathrm{~m}, y=1 \mathrm{~m})$ in the far field. And the corresponding sound pressure spectrum diagram is obtained, as shown in figure 12 .

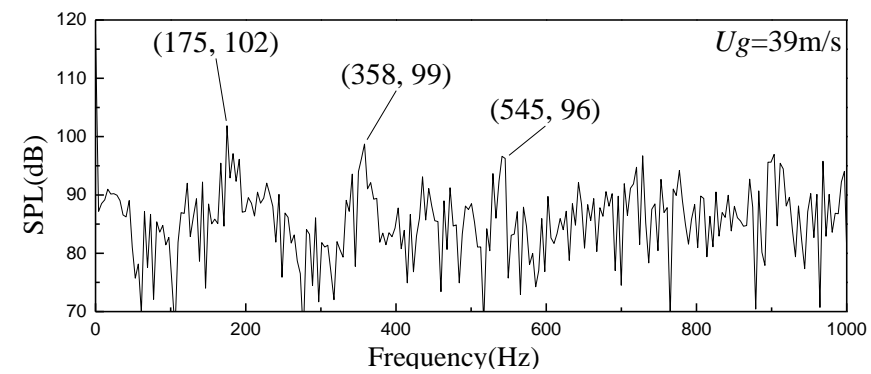

Figure 12 Sound Pressure Spectrum Calculation Result

As can be seen from the figure, at a frequency of 175 $\mathrm{Hz}$, there is a noise peak with a sound pressure level of 102 $\mathrm{dB}$. The higher harmonics can also be detected in the spectrum, with a maximum SPL of $102 \mathrm{~dB}$, corresponding to the second order harmonic component.

The sound pressure spectra at the velocities corresponding to figure 7 are given below $(37 \mathrm{~m} / \mathrm{s}, 38 \mathrm{~m} / \mathrm{s}$, $40 \mathrm{~m} / \mathrm{s}, 41 \mathrm{~m} / \mathrm{s}$ ), as shown in figure 13 .
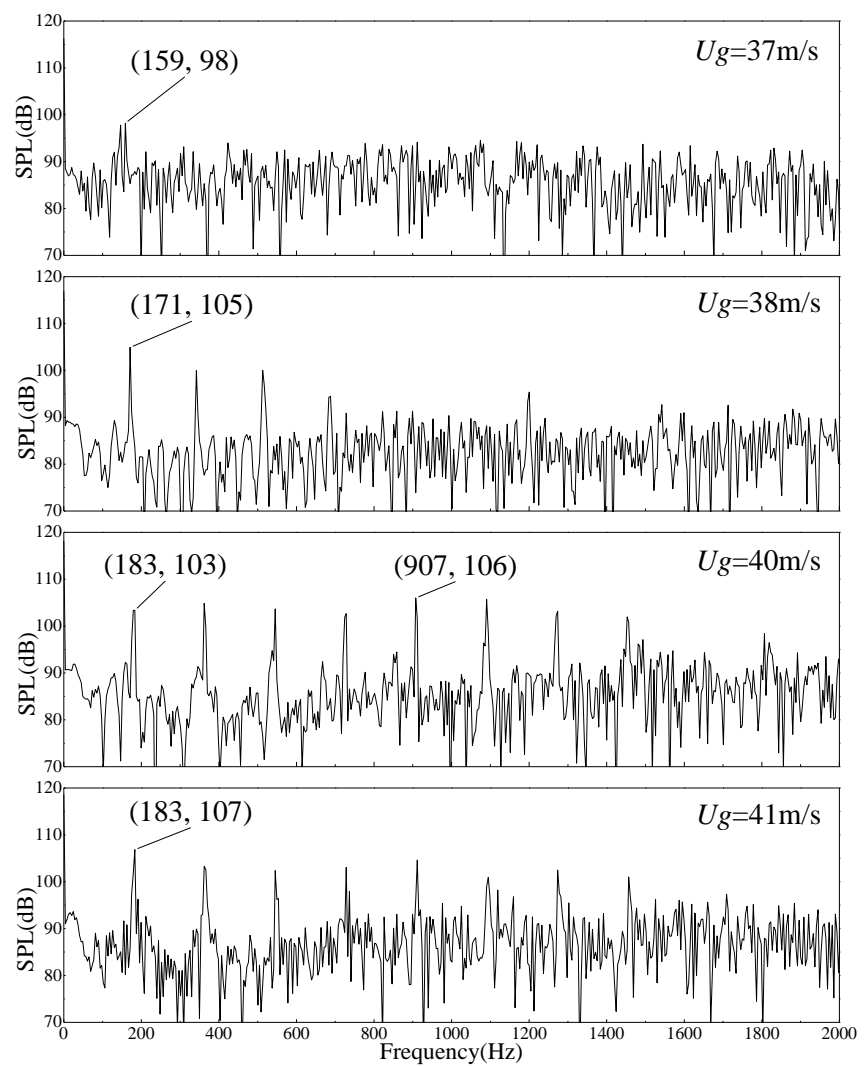

\section{Figure 13 Sound Pressure Spectrum Calculation Result}

The basic frequency component and the frequency corresponding to the maximum sound pressure level are illustrated in the above figure. It can be seen that within 2000 $\mathrm{Hz}$, there are peaks at integer multiple of the basic frequency, and the sound pressure value is substantially maintained at about 100-105 dB. Peaks also appear at non-multiplied frequencies.

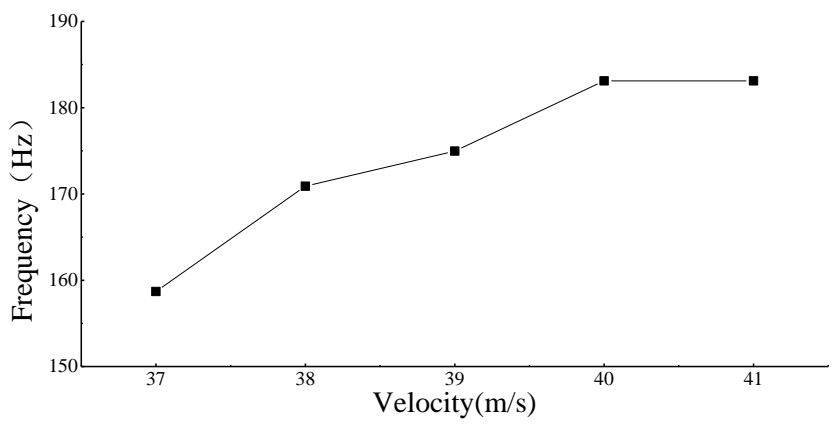

Figure 14 Sound Pressure Frequency Changes with the Flow Velocity

It can be seen that the sound pressure frequency and speed are basically linear. 


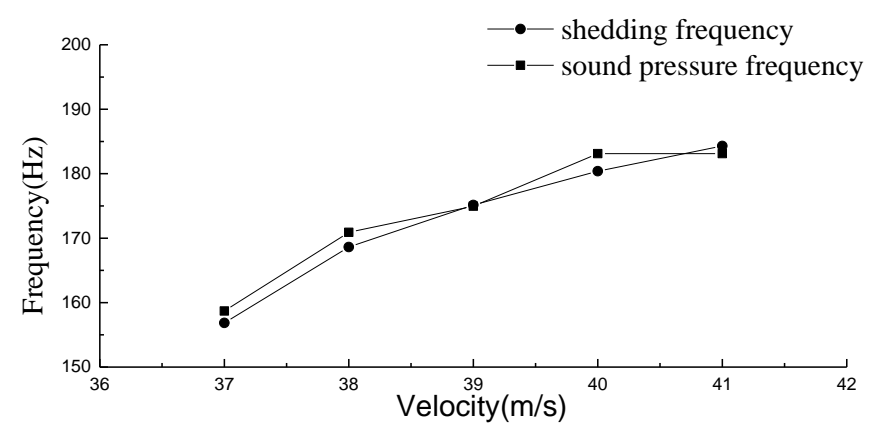

\section{Figure 15 the Frequency of the Falling Out of the Cavity Vortex Varies with the Frequency of the Sound Wave}

It can be seen that the sounding frequency of the cavity is consistent with the frequency of the cavity vortex shedding.

\section{CONCLUSIONS}

In this paper, the discrete vortex method is used to analyse the distribution of the flow field vortex in the cavity. Based on the Howe's vortex theory, the point vortex sound model of free space is established. The time domain boundary element method is used to simulate the scattering effect of the cavity wall on the sound, and the following conclusions are obtained:

(1) The discrete vortex method captures the process of cavity vortex from falling off, rolling up, downstream convection, and diffusion attenuation. The flow field in the cavity is characterized by large-scale flow oscillations accompanied by vortex generation and dissociation. This paper better explains the principle of cavity sounding caused by shear layer oscillation.

(2) The shedding frequencies at $37 \mathrm{~m} / \mathrm{s}, 38 \mathrm{~m} / \mathrm{s}, 39 \mathrm{~m} / \mathrm{s}$, $40 \mathrm{~m} / \mathrm{s}$ and $41 \mathrm{~m} / \mathrm{s}$ were calculated respectively. It was found that the frequency of shedding increased with the increase of velocity at low Mach numbers.

(3) The sound pressure frequencies of the far field at uniform flow with speed $37 \mathrm{~m} / \mathrm{s}, 38 \mathrm{~m} / \mathrm{s}, 39 \mathrm{~m} / \mathrm{s}, 40 \mathrm{~m} / \mathrm{s}$ and $41 \mathrm{~m} / \mathrm{s}$ are calculated. The sound pressure is basically around $100 \mathrm{~dB}$. It is found that in the range of $2000 \mathrm{~Hz}$, there are also peaks at the non-multiplied frequency. And although the sound pressure level is small, it embodies the complexity of the cavity noise.

After several numerical calculations, the author found that subtle changes in certain parameters will have a great impact on the results, and some results still need to be further improved. The ultimate purpose of the study of cavity flow mechanism is to grasp the mechanism of typical cavity flow pattern, and then provide theoretical support for the research of cavity noise reduction.

\section{NOMENCLATURE}

\section{Variables:}

$a$ Constant associated with the depth to length ratio of the cavity

c Sound speed

$d$ Depth of the cavity
$E(\cdot)$ Complete elliptical integral of the second kind

$E(\cdot \ \cdot)$ Incomplete elliptical integral of the second kind

$G$ Green's function

$H$ Heaviside function

$\boldsymbol{n}$ Out-of-boundary normal vector

$N$ Number of point vortices in the flow field

$p$ Pressure

$Q$ Field point

$R$ Distance between field point and source point

Re Reynolds number

$s$ Half the length of the cavity

$t$ Time

$u$ Velocity of the flow field

$v$ Airflow velocity

$W$ Velocity potential function

$z$ Complex coordinates in the physical domain

Subscripts:

$c_{s}$ Constant depends on the position of wave front

$S_{0}$ Sound source

$U_{g}$ Flow velocity

Greek:

$\alpha$ Constant related to the type of flow

$\gamma$ Single-point vortex source term

$\sigma$ Dirac delta function

$\lambda$ Complex coordinates in the transform domain

$v$ Kinematic viscosity coefficient

$\rho$ Airflow density

$\Gamma$ Strength of the point vortex

$\tau$ Delayed time

$\omega$ Rotation of the flow field

$\Pi$ Boundary of the calculation domain
Abbreviations:
DNS Direct numerical simulation
LES Large eddy simulation
FFT Fast Fourier Transformation

\section{ACKNOWLEDGMENTS}

The authors acknowledge financial support from the NSFC, under grant 51806146.

\section{References}

Abramowitz M., and Stegun I. A. (1964). Handbook of Mathematical Functions. National Bureau of Standards Publishing.

Allen R., and Mendonca F. (2004). DES validations of cavity acoustics over the subsonic to supersonic range. 10th AIAA/CEAS Aero Acoustics Conference. AIAA Paper, 2004-2862. doi: 10.2514/6.2004-2862.

Bilanin A. J., and Covert E. E. (2012). Estimation of possible excitation frequencies for shallow rectangular cavities. Aiaa Journal 11(3), 347-351. doi: 10.2514/3.6747.

Colonius T., Basu A. J., and Rowley C. W. (1999). Numerical investigation of the flow past a cavity. AIAA paper, 1999-1912. doi: 10.2514/6.1999-1912. 
Curle N.. (1955). The influence of solid boundaries upon aerodynamic sound. Proc.Roy.Soc. 231.doi: 10.1098/rspa.1955.0191.

Disselhorst J. H. M., and Wijngaarden L. V. (1980). Flow in the exit of open pipes during acoustic resonance. Journal of Fluid Mechanics Digital Archive, 99(02):27. doi: 10.1017/S0022112080000626.

Giesing J. P. (1969). Strength and kutta condition for unsteady multienergy flows. J Applied Mech 36(3). doi: $10.1115 / 1.3564724$.

Gloerfelt X. (2009). Cavity noise. VKI lecture series 3. doi: 10.1017/CBO9780511546143.014.

Gloerfelt X., Bogey C., and Bailly C. (2002). LES of the noise radiated by a flow over a rectangular cavity. ERCOFTAC Workshop on LES for Acoustics, 7-8.

Gloerfelt X., Bogey C., Bailly C., and Juve D. (2013). Aerodynamic noise induced by laminar and turbulent boundary layers over rectangular cavities. AIAA paper, 2002-2476. doi: 10.2514/6.2002-2476.

Grace S. M. (2001). An Overview of Computational Aeroacoustic Techniques Applied to Cavity Noise Prediction. AIAA, 0510.

Hardin J. C., and Mason J. P. (1977). Broadband noise generation by a vortex model of cavity flow. AIAA Journal, 15(5), 632-637. doi: 10.2514/3.60671.

Heller H. H., Holmes D. G., and Covert E. E. (1971). Flow-induced pressure oscillations in shallow cavities. Journal of sound and Vibration 18(4), 545-553. doi: 10.1016/0022-460X(71)90105-2.

Hong Z. L., Gao G., Jing X. D., and Sun X. F. (2015). A gird-less time domain method for plate trailing edge noise prediction. Acta Aeronautica et Astronautica Sinica 36(11), 3501-3514. doi: 10.7527/S1000-6893.2015.0008.

Howe M. S. (1999). Trailing edge noise at low mach numbers. Journal of Sound and Vibration 225(2), 211-238. doi: 10.1006/jsvi.1999.2236.

Howe M. S. (2003). Theory of vortex sound. Cambridge: Cambridge University Press.

Jing X., and Sun X. (2002). Sound-excited flow and acoustic nonlinearity at an orifice. Physics of Fluids 14(1), 268. doi: 10.1063/1.1423934.

Kiya M., Sasaki K., and Arie M. (1982). Discrete-vortex simulation of a turbulent separation bubble. Journal of Fluid Mechanics 120, 219-244.

Larchevêque L., Pierre S., and Labbé O. (2007). Largeeddy simulation of a subsonic cavity flow including asymmetric three-dimensional effects. Journal of Fluid Mechanics 577, 22. doi: 10.1017/S0022112006004502.

Mansur W. J. (1983). A time-stepping technique to solve wave propagation problems using the boundary element method. Southampton: University of Southampton.

Peters M. C. A. M., and Hirschberg A. (1993). Acoustically induced periodic vortex shedding at sharp edged open channel ends: simple vortex models. Journal of Sound and Vibration 161(2), 281-299. doi: 10.1006/jsvi.1993.1072.
Powell A. (1964). Theory of vortex sound. Journal of the Acoustical Society of America 36(1), 177-195. doi: 10.1121/1.1918931.

Rossiter J. E. (1964). Wind tunnel experiments on the flow over rectangular cavities at subsonic and transonic speeds. Ministry of Aviation; Royal Aircraft Establishment; RAE Farnborough.

Rott N. (1956). Diffraction of a weak shock with vortex generation. Journal of Fluid Mechanics 1, 111-128. doi: 10.1017/S0022112056000081.

Rowley C. W., Colonius T., and Basu A. J. (2002). On self-sustained oscillations in two-dimensional compressible flow over rectangular cavities. Journal of Fluid Mechanics 455, 315-346. doi: 10.1017/S0022112001007534.

Shieh C. M., and Morris P. J. (2013). Parallel numerical simulation of subsonic cavity noise. AIAA paper, 1999-1891. doi: 10.2514/6.1999-1891.

Tam C. K. W., and Block P. J. W. (1978). On the tones and pressure oscillations induced by flow over rectangular cavities. Journal of Fluid Mechanics 89(2), 373-399. doi: 10.1017/S0022112078002657.

Thompson D. J., Iglesias E. L., Liu X., Zhu J., and Hu Z. (2015). Recent developments in the prediction and control of aerodynamic noise from high-speed trains. International Journal of Rail Transportation 3(3), 119-150. doi: 10.1080/23248378.2015.1052996. 